Methods: The trial consisted of a double-blind, placebo-controlled First Period (16 weeks), in which adult patients with definite or probable DM (according to Bohan and Peter criteria) were randomized 1:1 to either high dose IVIg (2g/kg every 4 weeks) or placebo. Patients on placebo and patients without clinical worsening while on IVIg treatment entered the open label Extension Period (24 weeks) and received $2 \mathrm{~g} / \mathrm{kg}$ IVIg infusions every 4 weeks. To be included, subjects must have active disease with a manual muscle testing-8 (MMT-8) score $<142 / 150$. Patients who showed clinical worsening (defined according to Oddis et al, 2013 - with slight adaptation) at 2 consecutive visits between week 8 and week 16 were switched to the alternate treatment arm.

Primary endpoint was the proportion of responders in the IVIg vs. placebo arm at week 16, where response was defined per 2016 ACR/EULAR Myositis response criteria of at least minimal improvement [Total Improvement Score (TIS) $\geq 20$ points)] and without clinical worsening at 2 consecutive visits up to week 16 .

Results: A total of 95 adult DM patients (mean age: 53 years; $75 \%$ females; $92 \%$ Caucasian) were enrolled, with 47 and 48 randomized to IVIg and placebo, respectively. Baseline clinical characteristics (including medical history and prior DM medication) were balanced between the 2 arms.

The study met the primary endpoint at week 16, with the proportion of responders being significantly higher in the IVIg group (37/47; 78.7\%) as compared to the placebo group (21/48; 43.8\%; p-value 0.0008; Table 1).

Table 1. Total Improvement Score - Analysis of Proportion of Responders at Week 16 (Full Analysis Set, $\mathrm{N}=95$ )

\begin{tabular}{lccc}
\hline TIS Response & $\begin{array}{c}\text { octagam } 10 \% \\
\mathrm{~N}=\mathbf{4 7}\end{array}$ & $\begin{array}{c}\text { Placebo } \\
\mathrm{N}=\mathbf{4 8}\end{array}$ & $\begin{array}{c}\text { Difference octagam } 10 \% \\
\text { - placebo }\end{array}$ \\
\hline $\begin{array}{l}\text { Number }(\%) \text { of responders } \\
\text { Difference in response rates }\end{array}$ & $37(78.72 \%)$ & $21(43.75 \%)$ & \\
{$[95 \% \mathrm{Cl}] \mathrm{p}$-value ${ }^{\mathrm{a}}$} & & 34.97 \\
\hline
\end{tabular}

${ }^{a}$ Cochran-Mantel-Haenszel TestCl=confidence interval; $\mathrm{N}=$ number of patients; TIS=total improvement score

In the analysis of responders per improvement category at Week 16 , a $45.2 \%$ higher response rate for at least moderate improvement (TIS $\geq n 40$ points; $p<$ 0.0001 ) and a $23.6 \%$ higher response rate for at least major improvement (TIS $\geq 060$ points; $p<0.0062$ ) was observed in the IVIG group as compared to the placebo group.

The mean (SD) TIS was significantly higher in IVIg group [48.4 (24.4)] than in placebo arm [21.6 (20.2)] at week 16 (Fig 1).

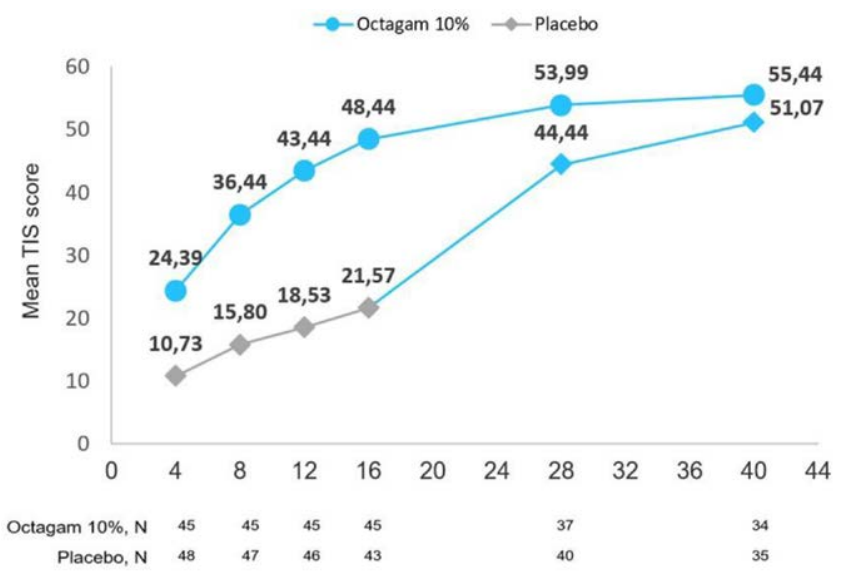

Figure 1.

After switching to IVIG in the Extension Period the placebo group attained a similar response rate at Week 40 as did the IVIg treated patients at Week 16, i.e approx. $70 \%$ for minimal improvement.

In line with the overall primary endpoint, secondary end points including all of the sub-components of TIS except muscle enzyme (MMT-8, MD global, Extramuscular global, patient global, HAQ,) as well as CDASI (Cutaneous Dermatomyositis Disease Area and Severity Index), also showed statistically significant improvement under IVIg treatment compared to placebo treatment.

The safety and tolerability profile of IVIg was consistent with previously reported safety outcomes for IVIg administration.

Conclusion: This is the first large international phase III randomized, placebo-controlled trial demonstrating the efficacy and safety of IVIg as a treatment for patients with DM.

REFERENCES:

[1] Oddis, C. V. et al. Arthritis Rheum (2013), 65, 314-324
Acknowledgements: Acknowledgments to all participating investigators, centers and patients and their families

Disclosure of Interests: Rohit Aggarwal Consultant of: Q32, Alexion, Argenx AstraZeneca, BMS, Boehringer Ingelheim, Corbus, Csl Behring, EMD Serono, Janssen, Kezar, Mallinckrodt, Kyverna, Octapharma, Orphazyme, Pfizer. Grant/research support from: BMS, Mallinckrodt, Pfizer, EMD Serono, Christina Charles-Schoeman Consultant of: Pfizer, Abbvie, Octapharma, Gilead, Regen eron-Sanofi, Grant/research support from: Bristol Myers Squibb, Pfizer, Abbvie, Octapharma, Joachim Schessl Speakers bureau: Octapharma, Grifols, CSL Behring, Consultant of: Octapharma, Zsuzsanna Bata-Csorgo Speakers bureau: Novartis, Sanofi-Genzyme, Ewopharma, Consultant of: Sanofi-Genzyme, Novartis, Ewopharma, Mazen Dimachkie Consultant of: ArgenX, Catalyst, Cello, CSL Behring, EcoR1, Kezar, Momenta, NuFactor, Octapharma, RaPharma/UCB, RMS Medical, Sanofi Genzyme, Shire Takeda, Spark Therapeutics and UCB Biopharma., Grant/research support from: Alexion, Alnylam Pharmaceuticals, Amicus, Biomarin, Bristol-Myers Squibb, Catalyst, Corbus, CSL-Behring, Glax oSmithKline, Genentech, Grifols, Kezar, Mitsubishi Tanabe Pharma, Novartis Octapharma, Orphazyme, Ra Pharma/UCB, Sanofi Genzyme, Sarepta Therapeutics, Shire Takeda, Spark Therapeutics, UCB Biopharma, Viromed/Healixmith., Zoltán Griger Speakers bureau: Abbvie, CSL-Behring, Eli-Lilly, Roche, Boehringer Ingelheim, Consultant of: Octapharma, Sergey Moiseev: None declared, Chester V Oddis Consultant of: EMD Serono; Alexion Pharmaceuticals, Inc, Grant/research support from: Genentech (Clinical trial support); Bristo Myers Squibb (Clinical trial support), Elena Schiopu Consultant of: Octapharma, Grant/research support from: Octapharma, Janssen (Johnson \& Johnson), BMS Pfizer, Abbvie, Jiří Vencovský Speakers bureau: Abbvie, Biogen, MSD, Pfizer, Roche, Sanofi, UCB, Consultant of: Abbvie, Boehringer, Eli Lilly, Octapharma, Gilead, Irene Beckmann Employee of: Octapharma, Todd Levine Shareholder of: Corinthian Reference Labs, CND Life Sciences, Consultant of: Grifols, Octapharma, Alexion, Elisabeth Clodi Employee of: Octapharma PPG, Vienna Austria, and the ProDERM Investigators: None declared DOI: 10.1136/annrheumdis-2021-eular.1389

\section{OP0009-PARE SUCCESSFUL PATIENT EDUCATION ON COVID-19 VACCINE SAFETY IN A LARGE RHEUMATOLOGY COHORT USING INTERACTIVE MOBILE-PHONE VIDEO TECHNOLOGY: CONTEXT, RESULTS, AND NEXT STEPS}

L. Rajagopala ${ }^{1}$, M. Ford ${ }^{1}$, M. Jasim ${ }^{1}$, D. Mulherin ${ }^{1}$, S. Venkatachalam¹, T. Sheeran ${ }^{1}$, J. Bateman ${ }^{1} .{ }^{1}$ The Rheumatology Centre, Cannock Wolverhampton, The Royal Wolverhampton NHS Trust,UK, Rheumatology, Wolverhampton, United Kingdom

Background: There are reported concerns of patient misunderstanding of the COVID-19 pandemic and vaccination safety. It is particularly important that these are understood in patients taking complex immunusuppressive therapies. Rapid delivery of targeted and up-to-date video messages from clinicians sent directly to patients could address patient uncertainty, and improve COVID-19 vaccination uptake. Innovative SMS (short message service) based video message has already shown promise in delivering COVID-19 information to patients [1] We present our experience in creating a tailored vaccine information video sent directly to our large cohort of patients in the UK with a range of rheumatic diseases and report on the learning points going forward.

Objectives: Our three objectives were: (1) educate our patients using an interactive mobile video information resource outlining the safety of the COVID vaccination in these patient groups; (2) better understand our patients' views of the COVID-19 vaccine; (3) evaluate the patient experience of this approach.

Methods: We designed and recorded an 8-minute interactive web-based video delivered education resource designed for mobile phones. This included: aims of the video; details of licenced vaccines; UK vaccination schedule; frequently asked questions; links to national charity resources; our clinician recommendations; a rheumatologist being vaccinated; case studies; and summary data. We produced a simple mobile phone web-based evaluation of the resource, including anonymised patient demographics, their understanding of the safety of the vaccine pre/ post video, and their user experience. Resource and evaluation were piloted by local healthcare professionals, our Patient Participation Group, a national charity, and approved by senior management. We distributed this to our follow-up patient cohort via our hospital SMS provider on 21.12.20, at the start of roll-out of UK vaccines, containing a link to the resource and evaluation.

Results: Of a cohort of 10,981 patients, we had 8886 mobile phone numbers. At Day 14, we recorded 2358 video views (26.3\%) and 664/2358 completed evaluations $(28.1 \%)$. Only one person reported being unable to watch the video. Before watching the video, 348/664 patients $(52.4 \%)$ were unsure if the vaccine was safe and/or recommended for them, rising to $626 / 664$ (94.3\%) post-video. Reasons for uncertainty after the video (38/664) included drug allergy and fertility concerns. Following the video, 509/664 patients $(76.6 \%)$ reported that they were more likely to have the vaccination. The majority of the patients $(614 / 660,93.0 \%)$ 
agreed that the method was a helpful method to share such information.Age distribution of our whole cohort, patients with mobile phones, and responders were similar: Age $>50$ was $80.4 \%, 76.3 \%$, and $88.6 \%$ respectively. A large proportion was treated with immunosuppressive medication $(61.3 \%$ conventional DMARD, $39.4 \%$ biological DMARD and $17.6 \%$ corticosteroids). Gender and case mix for responders were similar to published data from our cohort: female $74.0 \%$; rheumatoid arthritis commonest disease (389/664, 58.5\%).

Conclusion: To our knowledge, this is the first study to show the potential for SMS linked interactive multimedia message for patient education. The multimedia component allows users to easily navigate to relevant sections, and access a choice of linked resources. We demonstrate this low-cost technology is simple, effective and well placed to assist physicians in educating patients during a time when face-to-face contact is proving to be difficult. We have shown high levels of patient satisfaction, reassurance, and self-reported behaviour change. Such technology has potential utility for national bodies, primary and secondary care groups, and merits further research.

REFERENCES:

[1] The Lancet Rheumatology. Rapid distribution of information by SMS-embedded video link to patients during a pandemic. June 2020,Volume2,Number 6,e309-e368

Acknowledgements: We would like to thank the Hibbs Lupus Trust, and our Patient Participation Group.

Disclosure of Interests: None declared

DOI: 10.1136/annrheumdis-2021-eular.1778

\section{OP0010 EFFICACY AND SAFETY OF FAECAL MICROBIOTA TRANSPLANTATION FOR ACTIVE PERIPHERAL PSORIATIC ARTHRITIS: A RANDOMISED SHAM- CONTROLLED TRIAL}

M. Skov Kragsnaes ${ }^{1}$, J. Kjeldsen ${ }^{2}$, H. C. Horn ${ }^{1}$, H. L. Munk ${ }^{1}$, J. K. Pedersen ${ }^{3}$, S. A. Just ${ }^{3}$, P. Ahlquist ${ }^{4}$, F. Moeller Pedersen ${ }^{2}$, M. De Wit ${ }^{5}$, S. Möller ${ }^{6}$, V. Andersen ${ }^{7}$, K. Kristiansen ${ }^{8}$, H. M. Holt ${ }^{9}$, D. Kinggaard Holm ${ }^{10}$,

R. Christensen ${ }^{11}, \mathrm{~T}$. Ellingsen ${ }^{1}{ }^{1}$ Odense University Hospital, Department of Rheumatology, Odense, Denmark; ${ }^{2}$ Odense University Hospital, Department of Medical Gastroenterology, Odense, Denmark; ${ }^{3}$ Svendborg Hospital OUH, Section of Rheumatology, Department of Medicine, Svendborg, Denmark ${ }^{4}$ Reumaklinik Fyn, Reumaklinik Fyn, Odense, Denmark; ${ }^{5}$ Amsterdam, Patien Research Partner, Amsterdam, Netherlands; ${ }^{6}$ Odense University Hospital, OPEN - Open Patient data Explorative Network, Odense, Denmark; ${ }^{7}$ University Hospital of Southern Denmark, IRS-Centre Sønderjylland, Aabenraa, Denmark; ${ }^{8}$ University of Copenhagen, Laboratory of Genomics and Molecular Biomedicine, Department of Biology, Copenhagen, Denmark; ${ }^{9}$ Odense University Hospital, Department of Clinical Microbiology, Odense, Denmark; ${ }^{10}$ Odense University Hospital, Department of Clinical Immunology, Odense, Denmark; ${ }^{11}$ Bispebjerg and Frederiksberg Hospital, Musculoskeletal Statistics Unit, the Parker Institute, Copenhagen, Denmark

Background: Although causality remains to be established, targeting dysbiosis of the intestinal microbiota by faecal microbiota transplantation (FMT) has been proposed as a novel therapeutic option for treatment of extra-intestinal inflammatory diseases. ${ }^{1}$

Objectives: In this proof-of-concept study, we evaluated efficacy and safety of FMT in psoriatic arthritis (PsA). ${ }^{2}$

Methods: In this double-blind, parallel-group, sham-controlled, superiority trial, we randomly allocated (1:1) adults with active peripheral PsA ( $\geq 3$ swollen joints) despite ongoing treatment with methotrexate to one gastroscopic-guided FMT or sham transplantation into the duodenum. The transplants $(50 \mathrm{~g}$ faeces) came from one of four healthy, thoroughly screened, anonymous stool donors. 3 The primary efficacy endpoint was the proportion of participants experiencing treatment failure (i.e., needing treatment intensification) through 26 weeks. The first key secondary endpoint was change in Health Assessment Questionnaire Disability Index (HAQ-DI) score from baseline to week 26. Safety was monitored throughout the trial. Trial registration number: NCT03058900, ClinicalTrials.gov. Results: Of 97 screened, 31 (32\%) underwent randomisation (15 allocated to FMT), all received the assigned intervention, and $30(97 \%)$ completed the 26-week clinical evaluation (Table 1). Treatment failure occurred more frequently in the FMT group than in the sham group $(9[60 \%]$ vs $3[19 \%]$; risk ratio, 3.20; $95 \% \mathrm{Cl}, 1.06$ to $9.62 ; \mathrm{P}=0.018$ ). During the entire 26 weeks of observation, the rate of the treatment failures was significantly higher in the FMT than in the sham group, see figure 1. Improvement in HAQ-DI score differed between groups $(0.07$ vs 0.30 ) by 0.23 points $(95 \% \mathrm{Cl}, 0.02$ to $0.44 ; \mathrm{P}=0.031)$ in favour of sham. No serious adverse events were observed.

Conclusion: In this first interventional randomised controlled trial of FMT in immune-mediated arthritis, FMT was inferior to sham in treating active peripheral PsA. FMT did not appear to result in serious adverse events.

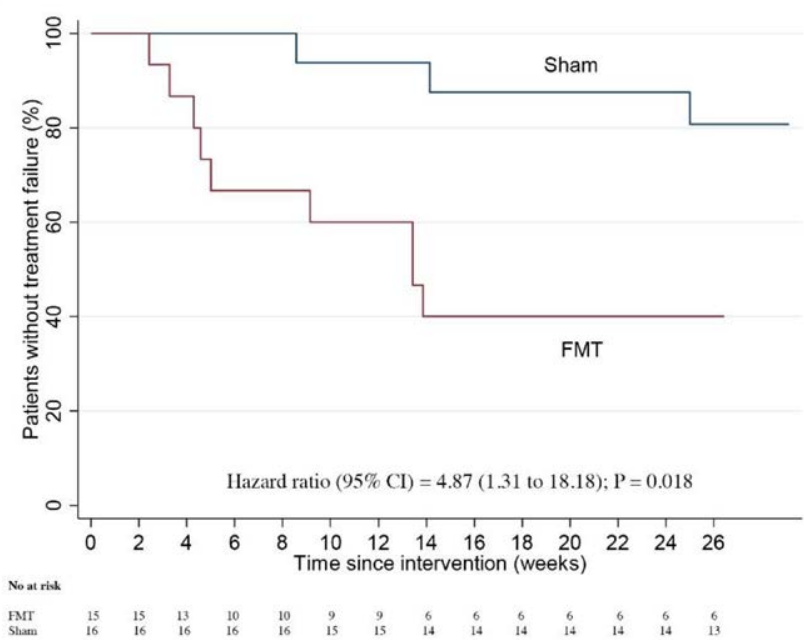

Figure 1. Time-to-event curves by intervention group from baseline to week 26.FMT, faecal microbiota transplantation.

Table 1. Baseline demographics and disease characteristics.

\begin{tabular}{lcc}
\hline \multicolumn{1}{c}{ Characteristic } & $\begin{array}{c}\text { FMT } \\
(\mathrm{n}=15)\end{array}$ & $\begin{array}{c}\text { Sham } \\
(\mathrm{n}=16)\end{array}$ \\
\hline Female sex, no. (\%) & $8(53 \%)$ & $12(75 \%)$ \\
Age, yr. & $48.9(16.1)$ & $52.4(11.0)$ \\
Height, cm & $175.2(7.0)$ & $169.8(8.6)$ \\
Weight, kg & $93.6(15.4)$ & $92.4(24.8)$ \\
Time since diagnosis, yr. ${ }^{\text {a }}$ & $2.6(0.3$ to 5.8$)$ & $5.6(0.5$ to 8.8$)$ \\
Rheumatoid factor IgM negative, no. $(\%)^{\mathrm{b}}$ & $13(93 \%)$ & $15(94 \%)$ \\
Anti-citrullinated peptide antibody negative, no. $(\%)^{\mathrm{b}}$ & $14(100 \%)$ & $16(100 \%)$ \\
HLA-B27 negative, no. (\%) & $15(100 \%)$ & $13(81 \%)$ \\
C-reactive protein, mg/L & $4.98(7.18)$ & $5.54(5.87)$ \\
HAQ-DI & $0.89(0.51)$ & $0.78(0.50)$ \\
Swollen joint 66 count & $7.5(3.0)$ & $6.7(2.7)$ \\
Tender joint 68 count & $14.9(8.9)$ & $17.3(8.8)$ \\
SPARCC enthesitis index & $13(87 \%)$ & $15(94 \%)$ \\
$\quad$ Score $\geq 1$, no. (\%) & $8.1(4.3)$ & $7.2(3.3)$ \\
Score in patients with a score $\geq 1$ &
\end{tabular}

Data are mean (SD) or $\mathrm{n}(\%)$ unless otherwise stated.FMT, faecal microbiota transplantation. ${ }^{a}$ Time since diagnosis of psoriatic arthritis is presented as median and interquartile range (IQR). ${ }^{\mathrm{b}}$ Presence of rheumatoid factor (IgM) and anti-citrullinated peptide antibody was not accessed in one patient from the FMT group.

\section{REFERENCES:}

[1] Manasson J, Blank RB, Scher JU. The microbiome in rheumatology: Where are we and where should we go? Ann Rheum Dis 2020;79:727-33.

[2] Kragsnaes MS, Kjeldsen J, Horn HC, et al. Efficacy and safety of faecal microbiota transplantation in patients with psoriatic arthritis: protocol for a 6-month, double-blind, randomised, placebo-controlled trial. BMJ Open 2018;8:e019231.

[3] Kragsnaes MS, Nilsson AC, Kjeldsen J, et al. How do I establish a stool bank for fecal microbiota transplantation within the blood- and tissue transplant service? Transfusion 2020;60:1135-41.

Acknowledgements: We thank all participants for their contribution. We thank CS Klinkby, trial nurse, for assistance in relation to the conduct of the trial visits. We also thank L Albjerg, biomedical laboratory technologist, AC Nilsson, consultant, KF Rasmussen, consultant, and J Georgsen, consultant, at the Department of Clinical Immunology, Odense University Hospital, Denmark, for assisting in the implementation of the FMT stool bank.

Disclosure of Interests: Maja Skov Kragsnaes Grant/research support from: Novartis 2017 (unrestricted research grant) to support 3 months PhD salary related to the conduct of the trial., Jens Kjeldsen: None declared, Hans Christian Horn: None declared, Heidi Lausten Munk: None declared, Jens Kristian Pedersen: None declared, Søren Andreas Just: None declared, Palle Ahlquist: None declared, Finn Moeller Pedersen: None declared, Maarten de Wit: None declared, Sören Möller: None declared, Vibeke Andersen: None declared, Karsten Kristiansen: None declared, Hanne Marie Holt: None declared, Dorte Kinggaard Holm: None declared, Robin Christensen: None declared, Torkell Ellingsen Grant/research support from: Novartis 2017 (unrestricted research grant)

DOI: 10.1136/annrheumdis-2021-eular.2046 